\title{
Convergence for stabilisation of degenerately convex minimisation problems
}

\author{
S. BARTELS $S^{\dagger}$ \\ Department of Mathematics, University of Maryland, College Park, MD 20742, USA \\ C. CARSTENSEN \\ Humboldt-Universität zu Berlin, Unter den Linden 6, 10099 Berlin, Germany \\ P. PLECHÁČ $\$$ \\ Mathematics Institute, University of Warwick, Coventry, CV4 7A, England
}

AND

A. $\mathrm{PROHL}^{\mathrm{II}}$

Department of Mathematics, ETHZ, CH-8092 Zurich, Switzerland

[Received 15 April 2003 and in revised form 6 March 2004]

\begin{abstract}
Degenerate variational problems often result from a relaxation technique in effective numerical simulation of nonconvex minimisation problems. The relaxed energy density is the convex envelope of the original one and so convex but not strictly convex. Hence strong convergence of straightforward finite element approximations cannot be expected but is relevant in many applications. This paper establishes a modified discretization by stabilisation and proves its convergence in strong norms.
\end{abstract}

Keywords: Degenerate variational problems; convexification; stabilisation; strong convergence; Euler-Lagrange equations; calculus of variations.

\section{Motivation and introduction}

The relaxation procedure in the calculus of variations allows the direct macroscopic simulation of models with finer and finer oscillations $[\mathrm{L} 1, \mathrm{~L} 2, \overline{\mathrm{BP}}]$. For the discrete problem this means that the nonconvex energy density is removed and replaced by some quasiconvex envelope or-in some applications - even the convex envelope; we refer to Example 1.1 for an illustration. The resulting discrete problem is then degenerate in the sense that it is convex but not strictly convex and so the Newton solver faces situations where the Hessian matrix for the tangential stiffness matrix is not positive definite and may be singular. Standard numerical regularisations are analysed in this paper as stabilisation techniques. Example 1.1 illustrates that the stabilisation allows less Newton iterations than the original relaxed problem. We prove for relevant examples that proper stabilisation maintains the convergence rates of the discrete problem, and, what came much to a surprise, yields even strong convergence of the strain variables in certain circumstances.

\footnotetext{
†'Email: sba@math.umd.edu

${ }^{\ddagger}$ Email: carstensen@math.hu-berlin.de

§Email: plechac@maths.warwick.ac.uk

II Email: apr@math.ethz.ch
} 
EXAMPLE 1.1 (3-Well Problem) Given $\Omega=(0,1)^{2}$ and boundary data $u_{D}(x)=v_{0}\left(x_{1}\right)+v_{0}\left(x_{2}\right)$ for $x=\left(x_{1}, x_{2}\right) \in \Omega$ and

$$
v_{0}(t)= \begin{cases}(t-1 / 4)^{3} / 6+(t-1 / 4) / 8 & \text { for } t \leqslant 1 / 4, \\ -(t-1 / 4)^{5} / 40-(t-1 / 4)^{3} / 8 & \text { for } t \geqslant 1 / 4\end{cases}
$$

the relaxation $W^{* *}$ (i.e. the lower convex envelope) of the 3-well energy density

$$
W(F)=\min \left\{|F|^{2},|F-(1,0)|^{2},|F-(0,1)|^{2}\right\}
$$

leads to the energy minimisation problem

$$
\begin{aligned}
\min _{u \in \mathcal{A}} E(u) \quad \text { for } \quad \mathcal{A}=\left\{v \in W^{1,2}(\Omega): v=u_{D} \text { on } \partial \Omega\right\} \quad \text { and } \\
E(u)=\int_{\Omega} W^{* *}(D u) \mathrm{d} x+\int_{\Omega}\left|u_{D}-u\right|^{2} \mathrm{~d} x+\int_{\Omega} f v \mathrm{~d} x
\end{aligned}
$$

with $f=\operatorname{div} D W^{* *}\left(D u_{D}\right)$. The exact solution of the relaxed minimisation problem reads $u(x)=u_{D}(x)$ for $x \in \Omega$. Its finite element approximation is computed on a sequence of uniform triangulations $\mathcal{T}$ of $\Omega$ with mesh size $h=1 / 2,1 / 4, \ldots, 1 / 32$ and degrees of freedom $N=1,9,49,225,961$ into triangles which are translated copies of $\operatorname{conv}\{(0,0),(0, h),(h, h)\}$ and $\operatorname{conv}\{(0,0),(h, h),(h, 0)\}$. Notice that $W^{* *}$ vanishes identically in $\operatorname{conv}\{(0,0),(1,0),(0,1)\} \subset \mathbb{R}^{2}$ and hence stabilisation is in order. The resulting discrete problem reads

$$
\min _{u_{h} \in \mathcal{A}_{h}} E_{h}\left(u_{h}\right) \quad \text { for } \quad E_{h}\left(u_{h}\right)=E\left(u_{h}\right)+h^{\gamma-1} \int_{\Omega}\left|D u_{h}\right|^{2} \mathrm{~d} x
$$

and $\mathcal{A}_{h}=\left\{v_{h} \in \mathcal{S}^{1}(\mathcal{T}): v_{h}=u_{D, h}\right.$ on $\left.\partial \Omega\right\}$ where $\mathcal{S}^{1}(\mathcal{T}) \subseteq W^{1,2}(\Omega)$ is the lowest order finite element space related to $\mathcal{T}$ and $u_{D, h}(z)=u_{D}(z)$ for all nodes $z$ on $\partial \Omega$.

For the exponents $\gamma=0,1 / 2,1,2$ and $\gamma=\infty\left(\gamma=\infty\right.$ means $E_{h}=E$, i.e. no stabilisation) we run a nested Newton-Raphson scheme. The termination criterion was an $\ell^{2}$ norm of the residual less than $10^{-9}$. Table 1 displays the history of iteration numbers $K$ as a function of $\gamma$ and $h$. This experimental result supports our strategy to approximate a degenerate convex problem by a slightly strictly convex one.

The paper is concerned with the convergence behaviour of the perturbed discrete solutions. The class of problems analysed in this paper is as follows. A natural finite element discretization of the Euler-Lagrange equations of a degenerately convex minimisation problem

$$
\text { (P) Seek } u \in \mathcal{A} \text { with } \int_{\Omega} S(D u): D v \mathrm{~d} x+J(u ; v)=0 \quad \text { for all } v \in \mathcal{A}_{D}
$$

(colon denotes the scalar product in $\mathbb{R}^{m \times n}$ ) with discrete spaces $\mathcal{A}_{h}=u_{D, h}+\mathcal{A}_{D, h}$ and $\mathcal{A}_{D, h} \subseteq \mathcal{A}_{D}$ reads

$$
\left(P_{h}\right) \quad \text { Seek } u_{h} \in \mathcal{A}_{h} \text { with } \int_{\Omega} S\left(D u_{h}\right): D v_{h} \mathrm{~d} x+J_{h}\left(u_{h} ; v_{h}\right)=0 \quad \text { for all } v_{h} \in \mathcal{A}_{D, h} .
$$

Typically, the nonlinear stress-strain function $S: \mathbb{R}^{m \times n} \rightarrow \mathbb{R}^{m \times n}$ is the derivative $S=D \varphi$ of an energy density function $\varphi$ that is (quasi-) convex but not strictly (quasi-)convex. Lacking 
TABLE 1

Iteration numbers $K$ required in the relaxed 3-well problem of Example 1.1 as a function of uniform mesh size $h$ and parameter $\gamma$. A minus sign means no convergence within 250 iteration steps.

\begin{tabular}{|l||c|c|c|c|c|}
\hline$h$ & $1 / 2$ & $1 / 4$ & $1 / 8$ & $1 / 16$ & $1 / 32$ \\
\hline \hline$\gamma=0$ & 4 & 4 & 5 & 7 & 8 \\
\hline$\gamma=1 / 2$ & 4 & 4 & 5 & 10 & 9 \\
\hline$\gamma=1$ & 4 & 4 & 5 & 13 & 16 \\
\hline$\gamma=2$ & 4 & 6 & 10 & 29 & - \\
\hline$\gamma=\infty$ & 4 & 10 & 98 & - & - \\
\hline
\end{tabular}

uniform convexity of $\varphi$ and so lacking uniform monotonicity of $S$ we cannot generally expect strong convergence of the error $e:=u-u_{h}$, namely

$$
\lim _{h \rightarrow 0}\|D e\|_{L^{p}(\Omega)}=0
$$

if an underlying mesh $\mathcal{T}_{h}$ becomes finer and finer such that the maximal mesh size tends to zero as $h \rightarrow 0$. Instead of 1.1 , one can merely expect weak convergence $D u_{h} \rightarrow D u$ in $L^{p}(\Omega)$ or convergence in weaker norms, e.g. $\lim _{h \rightarrow 0}\left\|u-u_{h}\right\|_{L^{r}(\Omega)}=0$. It turns out that the continuous lower order term $J: W^{1, p}\left(\Omega ; \mathbb{R}^{m}\right) \rightarrow W^{1, p}\left(\Omega ; \mathbb{R}^{m}\right)^{*}$ as well as boundary conditions in $\mathcal{A}:=\{v \in$ $W^{1, p}\left(\Omega ; \mathbb{R}^{m}\right): v=u_{D}$ on $\left.\Gamma_{D}\right\}$ for some part $\Gamma_{D}$ of the boundary $\partial \Omega$ of the domain $\Omega$ determine whether solutions $u$ or $u_{h}$ are unique or not; we refer to Section 2 for detailed assumptions. A typical time step in evolution of phase transitions leads to $(P)$ with an $L^{2}$-uniformly convex low-order term $J$ (see, e.g., [CP3] ) and requires strong convergence of gradients.

It is the aim of this paper to introduce stabilisation strategies to guarantee (1.1). For a meshdependent bilinear form $a_{h}: X_{h} \times Y_{h} \rightarrow \mathbb{R}$ such that $\mathcal{A}_{h} \subseteq X_{h}$ and $\mathcal{A}_{D, h} \subseteq Y_{h}$ we set $J_{h}\left(u_{h}, v_{h}\right):=J\left(u_{h}, v_{h}\right)+a_{h}\left(u_{h}, v_{h}\right)$. For relaxed nonconvex minimisation problems the additional term $a_{h}\left(u_{h}, v_{h}\right)$ allows a physical interpretation of a discrete surface energy. Provided that $(P)$ exhibits sufficient convexity, e.g. if $J$ is uniformly monotone with respect to an $L^{p}$ norm (on loworder terms) and $\varphi$ is convex, there exists a unique solution $u$ of $(P)$. Then if $u \in H^{3 / 2+\varepsilon}\left(\Omega ; \mathbb{R}^{m}\right)$ for some $\varepsilon>0$ we prove 1.1 for the unique discrete solution $u_{h}$ of $\left(P_{h}\right)$.

In order to illustrate some of the arguments in the proof of 1.1 we avoid in this introduction any technicalities through the (unrealistic) assumption $\mathcal{A}_{h}, \mathcal{A}_{D, h} \subseteq H^{2}\left(\Omega ; \mathbb{R}^{m}\right)$ and consider only one stabilisation term

$$
J_{h}\left(u_{h} ; v_{h}\right):=J\left(u_{h} ; v_{h}\right)+h^{2} \int_{\Omega} \Delta u_{h} \cdot \Delta v_{h} \mathrm{~d} x
$$

(dot denotes the scalar product in $\mathbb{R}^{m}$ ). Suppose furthermore that the low-order term $J$ is uniformly monotone such that standard arguments with the Galerkin orthogonality yield

$$
h^{2}\|\Delta e\|_{L^{2}(\Omega)}^{2}+\|e\|_{L^{2}(\Omega)}^{2} \leqslant C h^{2}
$$


for $u \in H^{2}\left(\Omega ; \mathbb{R}^{m}\right) \cap \mathcal{A}$. Then an integration by parts and $e=0$ on $\partial \Omega$ lead to

$$
\|D e\|_{L^{2}(\Omega)}^{2}=\int_{\Omega} D e: D e \mathrm{~d} x=-\int_{\Omega} e \cdot \Delta e \mathrm{~d} x .
$$

Cauchy's inequality, Young's inequality in the resulting upper bound, and 1.3 in the final step prove

$$
\|D e\|_{L^{2}(\Omega)}^{2} \leqslant\|e\|_{L^{2}(\Omega)}\|\Delta e\|_{L^{2}(\Omega)} \leqslant \frac{h}{2}\|\Delta e\|_{L^{2}(\Omega)}^{2}+\frac{h^{-1}}{2}\|e\|_{L^{2}(\Omega)}^{2} \leqslant C h .
$$

Hence we have strong convergence of gradients $(1.1)$ for $p=2$ if $u \in H^{2}\left(\Omega ; \mathbb{R}^{m}\right)$. Since this argument requires $C^{1}$ conforming finite elements the practical use of stabilisation (1.2) is limited. Therefore, this paper establishes three discrete stabilisations which lead to 1.1] in case $\mathcal{A}_{h}, \mathcal{A}_{D, h}$ are lowest order finite element spaces.

It should be stressed that stabilisation is in fact equivalent to that of [NW] stated for $m=n=1$ and for a numerical modification that replaces $J$ by a lumped version $J_{h}$. The proof of 1.11 in [NW] employs specific one-dimensional arguments for a particular model example. In contrast, stabilisation as introduced in this paper, and in $[\mathrm{P}]$ in the context of micromagnetism, appears to be a robust and flexible tool for a large class of degenerately convex minimisation problems. Convergence rates for the gradient error, however, require strong regularity conditions of the exact solution along with its uniqueness.

This paper is organised as follows. The general setting and the main results are presented in Section 2. A collection of examples for $S$ and $J$ that meet the abstract framework in $(P)$ are given in Section 3. In Section 4 we prove the main result. Notation and basic results related to finite element discretizations are introduced and recalled in Section 5. Sections 6-8 are devoted to three different stabilisations that define $\left(P_{h}\right)$ and lead to 1.1 via the abstract result of Section 2. Section 9 discusses strong convergence for a 2 -well problem which results from a model for phase transitions in crystalline solids. Numerical examples are reported in [Ba].

\section{General setting and main result}

This section is devoted to a general framework that allows several particular choices of the stabilisation term $a_{h}$ for a large class of examples indicated below. For this section, $J_{h}$ is quite general and could model a numerical quadrature for $J$ as well.

Given a bounded Lipschitz domain $\Omega \subset \mathbb{R}^{n}$ with polygonal (for $n=2$ ) or polyhedral (for $n=3$ ) boundary $\partial \Omega$ and $1<q \leqslant 2 \leqslant p<\infty, 1 / p+1 / q=1$. Given $u_{D} \in W^{1, p}\left(\Omega ; \mathbb{R}^{m}\right)$ set

$$
\mathcal{A}_{D}:=W_{0}^{1, p}\left(\Omega ; \mathbb{R}^{m}\right), \quad \mathcal{A}:=u_{D}+\mathcal{A}_{D}
$$

with $W_{0}^{1, p}\left(\Omega ; \mathbb{R}^{m}\right)=\left\{v \in W^{1, p}\left(\Omega ; \mathbb{R}^{m}\right):\left.v\right|_{\partial \Omega}=0\right\}$; moreover, let $|\cdot|_{W^{1, p}(\Omega)}$ denote the seminorm $|v|_{W^{1, p}(\Omega)}:=\|D v\|_{L^{p}(\Omega)}$ of $v \in W^{1, p}\left(\Omega ; \mathbb{R}^{m}\right)$. For a discrete space $\mathcal{A}_{D, h} \subseteq W_{0}^{1, p}\left(\Omega ; \mathbb{R}^{m}\right)$, spaces $X_{h}$ and $Y_{h}$, and an approximation $u_{D, h}$ of $u_{D}$ we merely suppose

$$
\mathcal{A}_{h}=u_{D, h}+\mathcal{A}_{D, h} \subseteq X_{h}, \quad \mathcal{A}_{D, h} \subseteq Y_{h} .
$$

The stress function $S: \mathbb{M}^{m \times n} \rightarrow \mathbb{R}$ and the low-order terms

$$
J: W^{1, p}\left(\Omega ; \mathbb{R}^{m}\right) \rightarrow\left(W^{1, p}\left(\Omega ; \mathbb{R}^{m}\right)\right)^{*}, \quad J_{h}: X_{h} \rightarrow Y_{h}^{*}
$$


of the continuous and discrete level, respectively, are supposed to satisfy the following hypotheses (H1)-(H3) for the exact and the discrete solution $u \in \mathcal{A}$ and $u_{h} \in \mathcal{A}_{h}$, respectively. A collection of examples follows below in Section 3

(H1) There exist positive constants $\alpha, r, s$ with $1<r \leqslant 2,0 \leqslant s<\infty$, and a function $S$ : $\mathbb{M}^{m \times n} \rightarrow \mathbb{M}^{m \times n}$ such that, for all $A, B \in \mathbb{M}^{m \times n}$,

$$
|S(A)-S(B)|^{r} \leqslant \alpha\left(1+|A|^{s}+|B|^{S}\right)(S(A)-S(B)):(A-B) .
$$

Here, $\mathbb{M}^{m \times n}$ denotes the real $m \times n$ matrices, and $|\cdot|$ the Frobenius norm related to the scalar product

$$
A: B=\sum_{k=1}^{m} \sum_{\ell=1}^{n} A_{k \ell} B_{k \ell} \quad \text { for } A, B \text { in } \mathbb{M}^{m \times n} .
$$

(H2) There exist solutions $u$ and $u_{h}$ of $(P)$ and $\left(P_{h}\right)$, respectively. [Their uniqueness is not assumed explicitly; at this stage, any choice will do. However, the uniqueness of $u$ is later a consequence of our strong regularity assumption.] That is, suppose that $u \in \mathcal{A}$ with $\sigma:=S(D u)$ and $u_{h} \in \mathcal{A}_{h}$ with $\sigma_{h}:=S\left(D u_{h}\right)$ satisfy

$$
\begin{array}{cc}
\int_{\Omega} \sigma: D v \mathrm{~d} x+J(u ; v)=0 & \text { for all } v \in \mathcal{A}_{D}, \\
\int_{\Omega} \sigma_{h}: D v_{h} \mathrm{~d} x+J_{h}\left(u_{h} ; v_{h}\right)=0 & \text { for all } v_{h} \in \mathcal{A}_{D, h} .
\end{array}
$$

Throughout this paper, set

$$
e:=u-u_{h}, \quad \delta:=\sigma-\sigma_{h} .
$$

(H3) There exist a constant $B>0$, a strictly convex function $\beta:[0, \infty) \rightarrow[0, \infty)$ with $\beta(0)=0$, and seminorms $\|\cdot\|_{X_{h}}$ and $\|\cdot\|_{Y_{h}}$ on the function spaces $X_{h}$ and $Y_{h}$ with $\mathcal{A}_{h} \subseteq X_{h} \subseteq$ $W^{1, p}\left(\Omega ; \mathbb{R}^{m}\right)$ and $\mathcal{A}_{D, h} \subseteq Y_{h} \subseteq W^{1, p}\left(\Omega ; \mathbb{R}^{m}\right)$ such that $e \in X_{h}, e-\mathcal{A}_{D, h} \subseteq Y_{h}$, and

$$
\begin{gathered}
\beta\left(\|e\|_{X_{h}}\right) \leqslant J_{h}(u ; e)-J_{h}\left(u_{h} ; e\right), \\
J_{h}(u, v)-J_{h}\left(u_{h} ; v\right) \leqslant B\|e\|_{X_{h}}\|v\|_{Y_{h}}
\end{gathered}
$$

for the exact and discrete solution $u$ and $u_{h}$ with the error $e=u-u_{h}$ from (H2), and $v \in e-\mathcal{A}_{D, h}$.

THEOREM 2.1 Suppose (H1)-(H3) hold and let $\beta^{*}$ denote the dual functional to $\beta$, i.e. $\beta^{*}(t)=$ $\sup \{s t-\beta(s): s \geqslant 0\}$. Then, for all $e_{h} \in \mathcal{A}_{D, h}$,

$$
\begin{aligned}
& (1-1 / r) \int_{\Omega} \delta: D e \mathrm{~d} x+\left(1 / c_{1}\right)\|\delta\|_{L^{q}(\Omega)}^{r}+\beta\left(\|e\|_{X_{h}}\right) \\
& \leqslant c_{2}\left|e-e_{h}\right|_{W^{1, p}(\Omega)}^{r /(r-1)}+\beta^{*}\left(2 B\left\|e-e_{h}\right\|_{Y_{h}}\right)+2\left(J_{h}\left(u ; e_{h}\right)-J\left(u ; e_{h}\right)\right) .
\end{aligned}
$$

The constants $c_{1}$ and $c_{2}$ depend on $\alpha, p, r, s$, and upper bounds for $|u|_{W^{1, p}(\Omega)}$ and $\left|u_{h}\right|_{W^{1, p}(\Omega)}$.

REMARK 2.1 It follows from (H1) that $0 \leqslant \delta: D e$ almost everywhere on $\Omega$; hence all the terms on the left-hand side in the estimate of the theorem are nonnegative. 
REMARK 2.2 It is known that $\beta^{*}:[0, \infty) \rightarrow[0, \infty)$ is a convex function with $\beta^{*}(0)=0$. In particular, for $\beta(t)=t^{2} / 2$ one finds $\beta^{*}(t)=t^{2} / 2$.

REMARK 2.3 The bounds of $|u|_{W^{1, p}(\Omega)}$ and $\left|u_{h}\right|_{W^{1, p}(\Omega)}$ may follow from further natural growth conditions on $S, J$, and $J_{h}$ which we have not stated here.

Throughout this paper we consider $J_{h}=\left.J\right|_{X_{h} \times Y_{h}}+a_{h}$ for a continuous bilinear form $a_{h}$ : $X_{h} \times Y_{h} \rightarrow \mathbb{R}$. Then we can replace (H3) by the following hypothesis.

(H4) Let $0<m \leqslant M<\infty$ satisfy

$$
m\|e\|_{L^{2}(\Omega)}^{2} \leqslant J(u ; e)-J\left(u_{h} ; e\right), \quad J(u ; v)-J\left(u_{h} ; v\right) \leqslant M\|e\|_{L^{2}(\Omega)}\|v\|_{L^{2}(\Omega)}
$$

for all $v \in W^{1, p}\left(\Omega ; \mathbb{R}^{m}\right)$.

Proposition 2.2 Suppose (H4) holds, and $\mathcal{A}_{h} \subseteq X_{h} \subseteq W^{1, p}\left(\Omega ; \mathbb{R}^{m}\right)$ and $\mathcal{A}_{D, h} \subseteq Y_{h} \subseteq$ $W^{1, p}\left(\Omega ; \mathbb{R}^{m}\right)$ are such that $e \in X_{h}$ and $e-\mathcal{A}_{D, h} \subseteq Y_{h}$. Assume that $a_{h}: X_{h} \times Y_{h} \rightarrow \mathbb{R}$ is a continuous bilinear form, $\|\cdot\|_{X_{h}}^{2}=\|\cdot\|_{Y_{h}}^{2}=\|\cdot\|_{L^{2}(\Omega)}^{2}+a_{h}(\cdot, \cdot)$, and $J_{h}=\left.J\right|_{X_{h} \times Y_{h}}+a_{h}$. Then (H3) holds with $\beta(t)=\min \{1, m\} t^{2}$ and $B:=\max \{1, M\}$.

Proof. This follows directly from the definitions of $J_{h},\|\cdot\|_{X_{h}},\|\cdot\|_{Y_{h}}$.

\section{Examples}

EXAMPLE 3.1 ( $p$-Laplacian) An energy minimisation of $|D u|^{p} / p$ leads to the $p$-Laplacian problem with $S(F)=|F|^{p-2} F$ and $2 \leqslant p<\infty$. Since (e.g. by a combination of Lemmas 2.1-2.3 in $[\overline{C K}]$ ) for any distinct $A, B \in \mathbb{R}^{n}$ and $\alpha=1+\max \{1, p-2\}^{2}$,

$$
\frac{|S(A)-S(B)|^{2}}{(S(A)-S(B)):(A-B)} \leqslant \alpha\left(|A|^{p-2}+|B|^{p-2}\right)
$$

it follows that (H1) is valid with $r=2, s=p-2$. See [CM, LB] for further results.

EXAMPLE 3.2 (Optimal Design) The relaxed model for an optimal design problem derived in [GKR] leads to a minimisation problem with energy density $\varphi(F)=\psi(|F|)$ and $S(F)=D \varphi(F)$. Given positive parameters $0<t_{1}<t_{2}$ and $0<\mu_{2}<\mu_{1}$ with $t_{1} \mu_{1}=t_{2} \mu_{2}$, the $C^{1}$ function $\psi:[0, \infty) \rightarrow[0, \infty)$ is defined by $\psi(0)=0$ and

$$
\psi^{\prime}(t)= \begin{cases}\mu_{1} t & \text { if } 0 \leqslant t \leqslant t_{1} \\ t_{1} \mu_{1}=t_{2} \mu_{2} & \text { if } t_{1} \leqslant t \leqslant t_{2} \\ \mu_{2} t & \text { if } t_{2} \leqslant t\end{cases}
$$

The function $S(F)$ satisfies (H1) with $r=2, s=0$, and $\alpha=\mu_{1}$ ([CP1]; cf. also [F]).

EXAMPLE 3.3 (Scalar 2-Well Problem) Given distinct wells $F_{1}, F_{2} \in \mathbb{R}^{n}$, the relaxed scalar 2-well problem leads to a convexified minimisation problem with energy density

$$
\varphi(F)=\max \left\{|F-B|^{2}-|A|^{2}, 0\right\}^{2}+4\left(|A|^{2}|F-B|^{2}-\left[A^{T}(F-B)\right]^{2}\right),
$$

where $A=\left(F_{2}-F_{1}\right) / 2$ and $B=\left(F_{1}+F_{2}\right) / 2$, and (H1) is satisfied with $r=2, s=2$, and $\alpha=4 \max \left\{2,\left|F_{1}-F_{2}\right|^{2}\right\}[\mathrm{CP} 1, \mathrm{~F}]$. This scalar problem can be deduced from the Ericksen-James energy density in an anti-plane shear model; the version for $n=1$, due to O. Bolza [Bo], serves as a master example in nonconvex minimisation [Y]. 
EXAMPLE 3.4 (Compatible Vectorial 2-Well Problem) Given two symmetric matrices $E_{1}, E_{2} \in$ $\mathbb{M}_{\text {sym }}^{n \times n}$, real numbers $W_{1}^{0}, W_{2}^{0} \in \mathbb{R}$, and a positive definite fourth order tensor $\mathbb{C}: \mathbb{M}_{\mathrm{sym}}^{n \times n} \rightarrow \mathbb{M}_{\mathrm{sym}}^{n \times n}$, let

$$
W_{j}(E)=\frac{1}{2}\left(E-E_{j}\right): \mathbb{C}\left(E-E_{j}\right)+W_{j}^{0}
$$

for $E \in \mathbb{M}_{\mathrm{sym}}^{n \times n}$ and $j=1,2$. Then if $E_{1}=E_{2}+(a \otimes b+b \otimes a) / 2$ for $a, b \in \mathbb{R}^{n}$ the quasiconvex hull of $W: \mathbb{M}_{\text {sym }}^{n \times n} \rightarrow \mathbb{R}, E \mapsto \min \left\{W_{1}(E), W_{2}(E)\right\}$, is convex and (see $[\mathbf{K}]$ ) given by

$$
\varphi(E)= \begin{cases}W_{1}(E) & \text { for } W_{2}(E)+\gamma \leqslant W_{1}(E), \\ \frac{1}{2}\left(W_{2}(E)+W_{1}(E)\right)-\frac{1}{4 \gamma}\left(W_{2}(E)-W_{1}(E)\right)^{2}-\frac{4}{\gamma} & \text { for }\left|W_{1}(E)-W_{2}(E)\right| \leqslant \gamma, \\ W_{2}(E) & \text { for } W_{1}(E)+\gamma \leqslant W_{2}(E),\end{cases}
$$

for $E \in \mathbb{M}_{\mathrm{sym}}^{n \times n}$ and $\gamma=\frac{1}{2}\left(E_{1}-E_{2}\right): \mathbb{C}\left(E_{1}-E_{2}\right)$. Then (H1) holds for $S(A)=D \varphi\left(\left(A+A^{T}\right) / 2\right)$, $A \in \mathbb{M}^{n \times n}$, with $r=2, s=0$, and a constant $0<\alpha$ that depends on $\mathbb{C}[$ [CP2] .

More physical examples in the context of nonconvex minimisation are included in [L1, L2, R].

EXAMPLE 3.5 (Linear Right-Hand Side) Given functions $f \in L^{q}\left(\Omega ; \mathbb{R}^{m}\right)$ and $g \in L^{q}\left(\Gamma_{N} ; \mathbb{R}^{m}\right)$ a typical linear right-hand side is, for $u, v \in W^{1, p}\left(\Omega ; \mathbb{R}^{m}\right)$,

$$
J(u ; v)=\int_{\Omega} f \cdot v \mathrm{~d} x+\int_{\Gamma_{N}} g \cdot v \mathrm{~d} s,
$$

where $\Gamma_{N}$ is a (possibly empty) part of $\partial \Omega$. Note that $J$ is independent of $u$ and hence does not satisfy (H4).

EXAMPLE 3.6 (Linear Low-Order Terms) The derivative $J=D \Psi$ of a strictly convex low-order term $\Psi$ in a model situation of [CP1] reads, for $u, v \in W^{1, p}\left(\Omega ; \mathbb{R}^{m}\right)$,

$$
J(u ; v)=\int_{\Omega} u \cdot v \mathrm{~d} s
$$

and satisfies (H4) for $m=M=1$.

\section{Proof of Theorem 2.1}

The proof of Theorem 2.1 extends a technique from [CP1]. From that paper we quote the first lemma.

LEMmA 4.1 Suppose (H1)-(H2) hold and $|\Omega|^{s / p}+|u|_{W^{1, p}(\Omega)}^{s}+\left|u_{h}\right|_{W^{1, p}(\Omega)}^{s} \leqslant c_{1} \alpha$. Then

$$
\|\delta\|_{L^{q}(\Omega)}^{r} \leqslant c_{1} \int_{\Omega} \delta: D e \mathrm{~d} x
$$

Proof. The proof follows (with different notation) the arguments that lead to formula (3.7) in [CP1] and is hence omitted.

Direct algebra and (H3) imply the following result. 
Lemma 4.2 Suppose (H2)-(H3) hold and $e_{h} \in \mathcal{A}_{D, h}$. Then

$$
\begin{gathered}
2 \int_{\Omega} \delta: D e \mathrm{~d} x+\beta\left(\|e\|_{X_{h}}\right) \leqslant \\
2 \int_{\Omega} \delta: D\left(e-e_{h}\right) \mathrm{d} x+\beta^{*}\left(2 B\left\|e-e_{h}\right\|_{Y_{h}}\right) \\
+2\left(J_{h}\left(u ; e_{h}\right)-J\left(u ; e_{h}\right)\right) .
\end{gathered}
$$

Proof. The two identities in (H2) with $v=e_{h}=v_{h} \in \mathcal{A}_{D, h} \subseteq \mathcal{A}_{D}$ yield

$$
\int_{\Omega} \delta: D e \mathrm{~d} x+J(u ; e)-J_{h}\left(u_{h} ; e\right)=\int_{\Omega} \delta: D\left(e-e_{h}\right) \mathrm{d} x+J\left(u ; e-e_{h}\right)-J_{h}\left(u_{h} ; e-e_{h}\right) .
$$

The differences on the left- and right-hand side are estimated by means of the first and second inequality of $(\mathrm{H} 3)$ after inserting $J_{h}(u ; e)$ and $J_{h}\left(u ; e-e_{h}\right)$, respectively, where $v=e-e_{h}$. Hence,

$$
\begin{aligned}
\int_{\Omega} \delta: D e \mathrm{~d} x+\beta\left(\|e\|_{X_{h}}\right)+\left(J(u ; e)-J_{h}(u ; e)\right) & \leqslant \int_{\Omega} \delta: D\left(e-e_{h}\right) \mathrm{d} x \\
& +B\|e\|_{X_{h}}\left\|e-e_{h}\right\|_{Y_{h}}+\left(J\left(u ; e-e_{h}\right)-J_{h}\left(u ; e-e_{h}\right)\right) .
\end{aligned}
$$

The definition of $\beta^{*}$ shows $s t \leqslant \beta(s)+\beta^{*}(t)$, which, for $s=\|e\|_{X_{h}}$ and $t=2 B\left\|e-e_{h}\right\|_{Y_{h}}$, results in

$$
2 B\|e\|_{X_{h}}\left\|e-e_{h}\right\|_{Y_{h}} \leqslant \beta\left(\|e\|_{X_{h}}\right)+\beta^{*}\left(2 B\left\|e-e_{h}\right\|_{Y_{h}}\right) .
$$

The combination of the last two estimates proves the lemma.

Lemma 4.3 Suppose (H1)-(H2) hold, and let $c_{2}:=2^{r^{\prime}} c_{1}^{r^{\prime}-1} / r^{\prime}$. Then

$$
2 \int_{\Omega} \delta: D\left(e-e_{h}\right) \mathrm{d} x \leqslant(1 / r) \int_{\Omega} \delta: D e \mathrm{~d} x+c_{2}\left|e-e_{h}\right|_{W^{1, p}(\Omega)}^{r^{\prime}}
$$

Proof. Hölder's and Young's inequalities show

$$
2 \int_{\Omega} \delta: D\left(e-e_{h}\right) \mathrm{d} x \leqslant\|\delta\|_{L^{q}(\Omega)}^{r} /\left(r c_{1}\right)+2^{r^{\prime}} c_{1}^{r^{\prime} / r}\left|e-e_{h}\right|_{W^{1, p}(\Omega)}^{r^{\prime}} / r^{\prime} .
$$

The assertion then follows from Lemma 4.1

Proof of Theorem 2.1. This follows from Lemmas 4.14 .3

\section{Finite element discretization}

Let $\mathcal{T}$ be a regular triangulation of $\Omega$ into triangles $(n=2)$ or tetrahedra $(n=3)$ in the sense of [BS], i.e. no hanging nodes, the domain is matched exactly, $\bar{\Omega}=\bigcup_{T \in \mathcal{T}} T$, and $\mathcal{T}$ satisfies the maximum angle condition. The extremal points of $T \in \mathcal{T}$ are called nodes and $\mathcal{N}$ denotes the set of all such nodes; $\mathcal{K}:=\mathcal{N} \backslash \partial \Omega$ is the subset of free nodes. The set of edges $(n=2)$ or faces $(n=3)$ $E=\operatorname{conv}\left\{z_{1}, \ldots, z_{n}\right\} \subseteq \partial T$ for pairwise distinct $z_{1}, \ldots, z_{n} \in \mathcal{N}$ and $T \in \mathcal{T}$ is denoted as $\mathcal{E}$. By $\mathcal{E}_{\Omega}$ we denote the set of interior edges or faces, $\mathcal{E}_{\Omega}=\left\{E \in \mathcal{E}: \exists T_{1}, T_{2} \in \mathcal{T}, E=T_{1} \cap T_{2}\right\}$. We assume that $\partial \Omega$ is matched exactly by edges on $\partial \Omega$, which implies $\partial \Omega=\bigcup_{E \in \mathcal{E}_{D}} E$ for the set of 
boundary edges $\mathcal{E}_{D}:=\{E \in \mathcal{E}: E \subseteq \partial \Omega\}$. Let $P_{k}(\omega)$ denote the set of algebraic polynomials of (total) degree $\leqslant k$ regarded as scalar functions on $\omega$. The set

$$
\mathcal{P}_{k}(\mathcal{T}):=\left\{v_{h} \in L^{\infty}(\Omega): \forall T \in \mathcal{T},\left.v_{h}\right|_{T} \in P_{k}(T)\right\}
$$

consists of all (possibly discontinuous) $\mathcal{T}$-elementwise polynomials of degree at most $k$. We define

$$
\mathcal{S}^{1}(\mathcal{T}):=\mathcal{P}_{1}(\mathcal{T}) \cap C(\bar{\Omega}) . \quad \mathcal{A}_{D, h}=\mathcal{S}_{0}^{1}(\mathcal{T})^{m}:=\mathcal{S}^{1}(\mathcal{T})^{m} \cap W_{0}^{1,2}\left(\Omega ; \mathbb{R}^{m}\right) .
$$

Supposing that $u_{D}$ is continuous on $\partial \Omega$ we choose $u_{D, h} \in \mathcal{S}^{1}(\mathcal{T})^{m}$ with $u_{D, h}(z)=u_{D}(z)$ for all $z \in \mathcal{N} \cap \partial \Omega$ and set

$$
\mathcal{A}_{h}:=u_{D, h}+\mathcal{S}_{0}^{1}(\mathcal{T})^{m} .
$$

Let $\left(\varphi_{z}: z \in \mathcal{N}\right)$ be the nodal basis of $\mathcal{S}^{1}(\mathcal{T})$, i.e. $\varphi_{z} \in \mathcal{S}^{1}(\mathcal{T})$ satisfies $\varphi_{z}(x)=0$ if $x \in \mathcal{N} \backslash\{z\}$ and $\varphi_{z}(z)=1$. We set $h_{T}:=\operatorname{diam}(T)$ for all $T \in \mathcal{T}$ and $h_{E}:=\operatorname{diam}(E)$ for all $E \in \mathcal{E}$ and define a function $h_{\mathcal{T}} \in \mathcal{L}^{0}(\mathcal{T})$ by $\left.h_{\mathcal{T}}\right|_{T}:=h_{T}$ for $T \in \mathcal{T}$. Abbreviate $h:=\left\|h_{\mathcal{T}}\right\|_{L^{\infty}(\Omega)}$. We will frequently assume that $\mathcal{T}$ is quasiuniform, which implies that $h \approx\left\|h_{\mathcal{T}}^{-1}\right\|_{L^{\infty}(\Omega)}^{-1}$.

We write $H^{s}\left(U ; \mathbb{R}^{m}\right)$ for $W^{s, 2}\left(U ; \mathbb{R}^{m}\right)$ for an open set $U \subseteq \mathbb{R}^{n}$ and

$$
H^{s}\left(\mathcal{T} ; \mathbb{R}^{m}\right)=\left\{v \in L^{2}\left(\Omega ; \mathbb{R}^{m}\right): \forall T \in \mathcal{T},\left.v\right|_{T} \in H^{s}\left(\operatorname{int}(T) ; \mathbb{R}^{m}\right)\right\} .
$$

The elementwise application of the differential operators $D^{2}$ (the matrix of all second order derivatives) and $\Delta$ (the Laplace operator) to a function $v \in H^{2}\left(\mathcal{T} ; \mathbb{R}^{m}\right)$ is denoted by $D_{\mathcal{T}}^{2} v$ and $\Delta_{\mathcal{T}} v$, respectively.

For each edge $E \in \mathcal{E}_{\Omega}$ we choose a vector $\nu_{E} \in \mathbb{R}^{n}$ (with selected and then fixed orientation) with $\left|v_{E}\right|=1$ orthogonal to $E$.

Assume $v \in H^{1}\left(\Omega ; \mathbb{R}^{m}\right) \cap H^{2}\left(\mathcal{T} ; \mathbb{R}^{m}\right)$, let $E \in \mathcal{E}_{\Omega}$ be such that $E=T_{+} \cap T_{-}$for $T_{+}, T_{-} \in \mathcal{T}$ and suppose $v_{E}$ points from $T_{+}$to $T_{-}$. Then define $[D v] \in L^{2}\left(E ; \mathbb{M}^{m \times n}\right)$ by

$$
[D v]:=\left.\left(\left.D v\right|_{T_{+}}\right)\right|_{E}-\left.\left(\left.D v\right|_{T_{-}}\right)\right|_{E} .
$$

For a function $\phi \in C\left(\partial \Omega ; \mathbb{R}^{m}\right)$ such that $\left.\phi\right|_{E} \in H^{2}\left(E ; \mathbb{R}^{m}\right)$ for all $E \in \mathcal{E}_{D}, \partial_{\mathcal{E}}^{2} \phi / \partial s^{2}$ is the edgewise second derivative of $\phi$ along $\partial \Omega ; H^{2}\left(\mathcal{E}_{D} ; \mathbb{R}^{m}\right)$ denotes the set of all such functions $\phi$.

Throughout this paper we abbreviate inequalities $A \leqslant C B$ with an $h$-independent constant $C>0$ by $A \lesssim B$, and $A \approx B$ replaces $A \lesssim B \lesssim A$. The constant $C$ may well depend on the shape of the elements; e.g. $h_{E} \approx h_{T}$ for $E \in \mathcal{E}$ and $T \in \mathcal{T}$ with $E \subseteq \partial T$. For instance, the well-established trace inequality reads

$$
\|\phi\|_{L^{2}(\partial T)}^{2} \lesssim h_{T}^{-1}\|\phi\|_{L^{2}(T)}^{2}+h_{T}\|D \phi\|_{L^{2}(T)}^{2}
$$

for any $T \in \mathcal{T}$ and $\phi \in H^{1}\left(T ; \mathbb{R}^{m}\right)$.

\section{Stabilisation via jumps of gradients}

This section is devoted to the discrete problem $\left(P_{h}\right)$ with $J_{h}:=J+a_{h}$ for the bilinear form

$$
a_{h}: X_{h} \times Y_{h} \rightarrow \mathbb{R}, \quad(v, w) \mapsto \sum_{E \in \mathcal{E}_{\Omega}} h_{E}^{\gamma} \int_{E}[D v]:[D w] \mathrm{d} s .
$$


Here, the spaces $X_{h}$ and $Y_{h}$ are arbitrary with

$$
X_{h}=Y_{h} \subseteq W^{1, p}\left(\Omega ; \mathbb{R}^{m}\right) \cap H^{3 / 2+\varepsilon}\left(\mathcal{T} ; \mathbb{R}^{m}\right)
$$

for some $\varepsilon$ with $0<\varepsilon \leqslant 1 / 2$. Then the traces of $D v$ and $D w$ on $\bigcup \mathcal{E}_{\Omega}$ for $v, w \in X_{h}=Y_{h}$ belong to $L^{2}\left(\bigcup \mathcal{E}_{\Omega}\right)$. Notice that $\mathcal{S}^{1}(\mathcal{T})^{m} \subseteq X_{h}$ but $e \in X_{h}$ is an additional (and strong) hypothesis on $u$, and that we will even suppose $u \in H^{2}\left(\Omega ; \mathbb{R}^{m}\right)$.

Theorem 6.1 Suppose $(\mathrm{H} 1),(\mathrm{H} 2)$, and $(\mathrm{H} 4)$ hold and $u_{D} \in H^{2}\left(\mathcal{E}_{D} ; \mathbb{R}^{m}\right)$. Moreover, assume that $u \in H^{2}\left(\Omega ; \mathbb{R}^{m}\right) \cap W^{1, p}\left(\Omega ; \mathbb{R}^{m}\right)$ and $\mathcal{T}$ is quasiuniform. Then

$$
\begin{gathered}
\lim _{h \rightarrow 0}\left\|D u-D u_{h}\right\|_{L^{2}(\Omega)}=0 \quad \text { for }-1<\gamma<3, \\
\left\|u-u_{h}\right\|_{W^{1,2}(\Omega)} \leqslant c_{3} h^{1 / 2} \quad \text { for } \gamma=1 .
\end{gathered}
$$

The constant $c_{3}>0$ depends on $c_{1}, c_{2}$, and upper bounds for $\|u\|_{H^{2}(\Omega)},\left|u_{h}\right|_{W^{1, p}(\Omega)},|u|_{W^{1, p}(\Omega)}$, and $\left\|\partial_{\mathcal{E}}^{2} u_{D} / \partial s^{2}\right\|_{L^{2}(\partial \Omega)}$.

REMARK 6.1 If $u \in\left(H^{2}\left(\mathcal{T} ; \mathbb{R}^{m}\right) \cap W^{1, p}\left(\Omega ; \mathbb{R}^{m}\right)\right) \backslash H^{2}\left(\Omega ; \mathbb{R}^{m}\right)$ then $a_{h}(u, \cdot) \not \equiv 0$. In that case, for $\gamma=5 / 2$, the proof of Theorem 6.1 below can be modified to obtain the estimate

$$
\left\|u-u_{h}\right\|_{W^{1,2}(\Omega)} \lesssim h^{1 / 8}
$$

The proof of the theorem follows from the abstract estimate of Theorem 2.1 and the following lemmas. Throughout this section, abbreviate

$$
|v|_{h}:=\left\|h_{\mathcal{E}}^{\gamma / 2}[D v]\right\|_{L^{2}\left(\bigcup \mathcal{E}_{\Omega}\right)}, \quad\|v\|_{X_{h}}^{2}=\|v\|_{Y_{h}}^{2}:=\|v\|_{L^{2}(\Omega)}^{2}+|v|_{h}^{2}
$$

for $v \in H^{3 / 2+\varepsilon}\left(\mathcal{T} ; \mathbb{R}^{m}\right)$.

LEMMA 6.1 If $e_{h}$ is the nodal interpolant of $e \in C\left(\bar{\Omega} ; \mathbb{R}^{m}\right)$ then

$$
\left\|e-e_{h}\right\|_{Y_{h}} \lesssim\left\|h_{\mathcal{T}}^{(1+\gamma) / 2} D_{\mathcal{T}}^{2} e\right\|_{L^{2}(\Omega)}
$$

Proof. This is an immediate consequence of the trace inequality (5.1) and standard error estimates of nodal interpolation.

Proposition 2.2 and Lemma 6.1 allow for the application of Theorem 2.1 The strong convergence, however, is obtained by a combination with the following argument.

LEMMA 6.2 We have

$$
\begin{aligned}
|e|_{W^{1,2}(\Omega)}^{2} \lesssim & \|e\|_{L^{2}(\Omega)}\left\|\Delta_{\mathcal{T}} e\right\|_{L^{2}(\Omega)}+|e|_{h}\left(\left\|h_{\mathcal{T}}^{(1-\gamma) / 2} D e\right\|_{L^{2}(\Omega)}+\left\|h_{\mathcal{T}}^{-(1+\gamma) / 2} e\right\|_{L^{2}(\Omega)}\right) \\
& +\left\|h_{\mathcal{T}}^{2} \partial_{\mathcal{E}}^{2} u_{D} / \partial s^{2}\right\|_{L^{2}(\partial \Omega)}\left(\|u\|_{H^{2}(\Omega)}+\left\|h_{\mathcal{T}}^{-1 / 2} D u_{h}\right\|_{L^{2}(\Omega)}\right)
\end{aligned}
$$

Proof. We perform an integration by parts on each $T \in \mathcal{T}$, use the estimates $\|D u \cdot v\|_{L^{2}(\partial \Omega)} \lesssim$ 
$\|u\|_{H^{2}(\Omega)}$ and $\left\|D u_{h} \cdot v\right\|_{L^{2}(\partial \Omega)} \lesssim\left\|h_{\mathcal{T}}^{-1 / 2} D u_{h}\right\|_{L^{2}(\Omega)}$, and employ the Cauchy inequalities to verify

$$
\begin{aligned}
\|D e\|_{L^{2}(\Omega)}^{2}= & \sum_{T \in \mathcal{T}} \int_{\partial T}(D e \cdot v) \cdot e \mathrm{~d} s-\sum_{T \in \mathcal{T}} \int_{T}(\Delta e) \cdot e \mathrm{~d} x \\
= & \sum_{E \in \mathcal{E}_{\Omega}} \int_{E}\left([D e] \cdot v_{E}\right) \cdot e \mathrm{~d} s-\int_{\Omega}\left(\Delta_{\mathcal{T}} e\right) \cdot e \mathrm{~d} x+\int_{\partial \Omega}(D e \cdot v) \cdot e \mathrm{~d} s \\
\lesssim & \left(\sum_{E \in \mathcal{E}_{\Omega}} h_{E}^{\gamma}\|[D e]\|_{L^{2}(E)}^{2}\right)^{1 / 2}\left(\sum_{E \in \mathcal{E}_{\Omega}} h_{E}^{-\gamma}\|e\|_{L^{2}(E)}^{2}\right)^{1 / 2} \\
& +\left\|\Delta_{\mathcal{T}} e\right\|_{L^{2}(\Omega)}\|e\|_{L^{2}(\Omega)}+\left(\|u\|_{H^{2}(\Omega)}+\left\|h_{\mathcal{T}}^{-1 / 2} D u_{h}\right\|_{L^{2}(\Omega)}\right)\|e\|_{L^{2}(\partial \Omega)} \\
= & |e|_{h}\left(\sum_{E \in \mathcal{E}_{\Omega}} h_{E}^{-\gamma}\|e\|_{L^{2}(E)}^{2}\right)^{1 / 2}+\|e\|_{L^{2}(\Omega)}\left\|\Delta_{\mathcal{T}} e\right\|_{L^{2}(\Omega)} \\
& +\left(\|u\|_{H^{2}(\Omega)}+\left\|h_{\mathcal{T}}^{-1 / 2} D u_{h}\right\|_{L^{2}(\Omega)}\right)\|e\|_{L^{2}(\partial \Omega)} .
\end{aligned}
$$

The trace inequality (5.1) yields

$$
\sum_{E \in \mathcal{E}_{\Omega}} h_{E}^{-\gamma}\|e\|_{L^{2}(E)}^{2} \lesssim\left\|h_{\mathcal{T}}^{-(1+\gamma) / 2} e\right\|_{L^{2}(\Omega)}^{2}+\left\|h_{\mathcal{T}}^{(1-\gamma) / 2} D e\right\|_{L^{2}(\Omega)}^{2} .
$$

Nodal interpolation estimates on each $E \in \mathcal{E}_{D}$ show

$$
\|e\|_{L^{2}(\partial \Omega)} \lesssim\left\|h_{\mathcal{T}}^{2} \partial_{\mathcal{E}}^{2} u_{D} / \partial s^{2}\right\|_{L^{2}(\partial \Omega)} .
$$

The combination of the last three estimates concludes the proof.

Proof of Theorem 6.1] Notice that $\left.[D u]\right|_{E}=0$ for all $E \in \mathcal{E}_{\Omega}$ so that $a_{h}\left(u, e_{h}\right)=0$. It follows from Theorem 2.1 and Lemma6.1 that

$$
\begin{aligned}
\|e\|_{L^{2}(\Omega)}^{2}+|e|_{h}^{2} & \lesssim\left|e-e_{h}\right|_{W^{1, p}(\Omega)}^{r /(r-1)}+\beta^{*}\left(2 B\left\|e-e_{h}\right\|_{Y_{h}}\right)+2 a_{h}\left(u, e_{h}\right) \\
& \lesssim\left\|h_{\mathcal{T}} D_{\mathcal{T}}^{2} e\right\|_{L^{p}(\Omega)}^{r /(r-1)}+\left\|h_{\mathcal{T}}^{(\gamma+1) / 2} D_{\mathcal{T}}^{2} e\right\|_{L^{2}(\Omega)}^{2} \lesssim h^{r /(r-1)}+h^{\gamma+1}=: \mathrm{RHS}^{2} .
\end{aligned}
$$

The combination of this with Lemma 6.2 and $\left\|\Delta_{\mathcal{T}} e\right\|_{L^{2}(\Omega)},\|u\|_{H^{2}(\Omega)},\left\|\partial_{\mathcal{E}}^{2} u_{D} / \partial s^{2}\right\|_{L^{2}(\partial \Omega)}$, $\left\|D u_{h}\right\|_{L^{2}(\Omega)} \lesssim 1$ yields

$$
\begin{aligned}
|e|_{W^{1,2}(\Omega)}^{2} \lesssim & \operatorname{RHS}+\left\|h_{\mathcal{T}}^{2}\right\|_{L^{\infty}(\Omega)}\left\|h_{\mathcal{T}}^{-1 / 2}\right\|_{L^{\infty}(\Omega)} \\
& +\operatorname{RHS}\left(\left\|h_{\mathcal{T}}^{-(1+\gamma) / 2}\right\|_{L^{\infty}(\Omega)} \operatorname{RHS}+\left\|h_{\mathcal{T}}^{(1-\gamma) / 2}\right\|_{L^{\infty}(\Omega)}\|D e\|_{L^{2}(\Omega)}\right) .
\end{aligned}
$$

Young's inequality allows us to absorb $\|D e\|_{L^{2}(\Omega)}=|e|_{W^{1,2}(\Omega)}$ on the right-hand side and hence shows

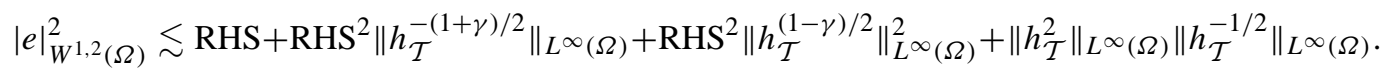

Since $r \leqslant 2, h^{r /(r-1)} \lesssim h^{2}$. From $\left\|h_{\mathcal{T}}^{-1}\right\|_{L^{\infty}(\Omega)} \approx\left\|h_{\mathcal{T}}\right\|_{L^{\infty}(\Omega)}^{-1}$ we deduce

$$
|e|_{W^{1,2}(\Omega)}^{2} \lesssim h+h^{(\gamma+1) / 2}+h^{(3-\gamma) / 2}+h^{(\gamma+1) / 2}+h^{3-\gamma}+h^{2}+h^{3 / 2} .
$$

This and $\|e\|_{L^{2}(\Omega)} \lesssim h^{2}+h^{\gamma+1}$ prove Theorem 6.1. 
REMARK 6.2 If boundary conditions are imposed only on some part $\Gamma_{D}$ of $\partial \Omega$ (and not on the entire boundary $\partial \Omega$ ) one obtains an additional term

$$
\int_{\partial \Omega \backslash \Gamma_{D}}(D e \cdot v) \cdot e \mathrm{~d} s
$$

which we have been unable to control.

\section{Stabilisation via distances to averages of gradients}

This section is devoted to a stabilisation $J_{h}=J+a_{h}$ with distances to averages of gradients, i.e.

$$
a_{h}(v, w):=\int_{\Omega} h_{\mathcal{T}}^{\gamma-1}(D v-A D v):(D w-A D w) \mathrm{d} x
$$

for $\gamma \in \mathbb{R}, v, w \in W^{1, p}\left(\Omega ; \mathbb{R}^{m}\right)$, and for the averaging operator

$$
A: L^{2}\left(\Omega ; \mathbb{M}^{m \times n}\right) \rightarrow \mathcal{S}^{1}(\mathcal{T})^{m \times n}, \quad p \mapsto A p:=\sum_{z \in \mathcal{N}}\left|\omega_{z}\right|^{-1} \int_{\omega_{z}} p \mathrm{~d} x \varphi_{z} .
$$

Here, for each node $z \in \mathcal{N}, \omega_{z}=\left\{x \in \Omega: \varphi_{z}(x)>0\right\}$ denotes its patch of area or volume $\left|\omega_{z}\right|$. Let $X_{h}=Y_{h}$ be as in Section6. For $v \in X_{h}$ we abbreviate

$$
\|v \mid\|_{h}^{2}=a_{h}(v, v)=\left\|h_{\mathcal{T}}^{(\gamma-1) / 2}(D v-A D v)\right\|_{L^{2}(\Omega)}^{2}
$$

and define $\|\cdot\|_{X_{h}}=\|\cdot\|_{Y_{h}}$ by

$$
\|v\|_{X_{h}}^{2}=\|v\|_{Y_{h}}^{2}=\|v\|_{L^{2}(\Omega)}^{2}+\|\| v \|_{h}^{2} .
$$

THEOREM 7.1 Under the hypotheses of Theorem 6.1.

$$
\begin{array}{cc}
\lim _{h \rightarrow 0}\left\|D u-D u_{h}\right\|_{L^{2}(\Omega)}=0 & \text { for }-1<\gamma<3, \\
\left\|u-u_{h}\right\|_{W^{1,2}(\Omega)} \leqslant c_{4} h^{1 / 2} & \text { for } \gamma=1 .
\end{array}
$$

REMARK 7.1 Provided $u \in\left(H^{2}\left(\mathcal{T} ; \mathbb{R}^{m}\right) \cap W^{1, p}\left(\Omega ; \mathbb{R}^{m}\right)\right) \backslash H^{2}\left(\Omega ; \mathbb{R}^{m}\right)$ and $\gamma=5 / 2$, one can prove

$$
\left\|u-u_{h}\right\|_{W^{1,2}(\Omega)} \lesssim h^{1 / 8} .
$$

The following lemma shows that the stabilisation defined by 7.1 is equivalent to the one discussed in the previous section and will be used to reduce the proof of Theorem 7.1 to the one of Theorem 6.1. The seminorm $|\cdot|_{h}$ is defined as in the previous section.

LEMMA $7.1\left([\overline{\mathrm{C}} \mid)\right.$ For $v_{h} \in \mathcal{S}^{1}(\mathcal{T})^{m}$ we have $\left|v_{h}\right|_{h} \approx\left\|\mid v_{h}\right\|_{h}$.

Proof of Theorem 7.1. Let $e_{h}$ denote the nodal interpolant of $e \in C\left(\bar{\Omega} ; \mathbb{R}^{m}\right)$. Theorem 2.1 and Proposition 2.2 show

$$
\|e\|_{L^{2}(\Omega)}^{2}+\left\|\left|e\left\|_{h}^{2} \lesssim\left|e-e_{h}\right|_{W^{1, p}(\Omega)}^{r /(r-1)}+\right\| e-e_{h}\left\|_{L^{2}(\Omega)}^{2}+\right\| e-e_{h} \|_{h}^{2}+2 a_{h}\left(u, e_{h}\right) .\right.\right.
$$


Lemma 7.1 shows

$$
|e|_{h} \leqslant\left|e_{h}\right|_{h}+\left.\left|e-e_{h}\right|_{h} \lesssim||\left|e_{h}\right|\right|_{h}+\left|e-e_{h}\right|_{h} \lesssim\|||\|_{h}+\left|\left\|e-e_{h}\left|\|_{h}+\right| e-\left.e_{h}\right|_{h} .\right.\right.
$$

Nodal interpolation estimates and continuity of $A$ then imply

$$
|e|_{h}^{2} \lesssim\left\|\left|e_{h}\right|\right\|_{h}^{2}+h^{\gamma+1}
$$

We employ Hölder's inequality, Young's inequality, and nodal interpolation estimates to verify that for $\varrho>0$,

$$
a_{h}\left(u, e_{h}\right) \lesssim\|u\|\left\|_{h}^{2}+\varrho\right\|\left\|e_{h}\right\|_{h}^{2} \lesssim\|\| u\left\|_{h}^{2}+\varrho\right\|\left|e\left\|_{h}^{2}+\varrho\right\|\right| e-e_{h}\left\|_{h}^{2} \lesssim\right\|\|u\|_{h}^{2}+\varrho\|\mid\|_{h}^{2}+h^{\gamma+1} .
$$

Using $\sum_{z \in \mathcal{N}} \varphi_{z}=1$, we deduce

$$
\begin{aligned}
\|u\|_{h}^{2} & =\sum_{z \in \mathcal{N}} \int_{\Omega} h_{\mathcal{T}}^{\gamma-1} \varphi_{z}\left(D u-p_{z}\right)(D u-A D u) \mathrm{d} x \\
& \leqslant\left(\sum_{z \in \mathcal{N}}\left\|h_{\mathcal{T}}^{(\gamma-1) / 2} \varphi_{z}^{1 / 2}\left(D u-p_{z}\right)\right\|_{L^{2}(\Omega)}^{2}\right)^{1 / 2}\left\|h_{\mathcal{T}}^{(\gamma-1) / 2}(D u-A D u)\right\|_{L^{2}(\Omega)},
\end{aligned}
$$

where $p_{z}=\left|\omega_{z}\right|^{-1} \int_{\omega_{z}} D u \mathrm{~d} x$ for all $z \in \mathcal{N}$. Poincaré's inequality and $\left|\varphi_{z}\right| \leqslant 1$ show

$$
\sum_{z \in \mathcal{N}}\left\|h_{\mathcal{T}}^{(\gamma-1) / 2} \varphi_{z}^{1 / 2}\left(D u-p_{z}\right)\right\|_{L^{2}(\Omega)}^{2} \lesssim\left\|h_{\mathcal{T}}^{(\gamma+1) / 2} D^{2} u\right\|_{L^{2}(\Omega)}^{2}
$$

The combination of the preceding three estimates proves

$$
\|e\|_{L^{2}(\Omega)}^{2}+|e|_{h}^{2} \lesssim h^{2}+h^{\gamma+1} .
$$

The assertions of the theorem then follow from Lemma 6.2 and the arguments of the proof of Theorem6.1.

\section{Stabilisation via gradients}

This section is devoted to a stabilisation $J_{h}=J+a_{h}$ with gradients, i.e. with

$$
a_{h}(v, w)=h^{\gamma} \int_{\Omega} D v: D w \mathrm{~d} x
$$

for some $\gamma>0$ and all $v, w \in X_{h}=Y_{h}=W^{1, p}\left(\Omega ; \mathbb{R}^{m}\right)$. For $v \in X_{h}$ we define

$$
\|v\|_{X_{h}}^{2}=\|v\|_{Y_{h}}^{2}:=\|v\|_{L^{2}(\Omega)}^{2}+h^{\gamma}\|D v\|_{L^{2}(\Omega)}^{2} .
$$

TheOREM 8.1 Suppose (H1), (H2), and (H4) hold. Assume that $\mathcal{T}$ is quasiuniform and $u \in$ $W^{1, p}\left(\Omega ; \mathbb{R}^{m}\right) \cap H^{1+s}\left(\Omega ; \mathbb{R}^{m}\right)$ for some $s \in(1 / 2,1]$. Then

$$
\begin{array}{ll}
\lim _{h \rightarrow 0}\left\|D u-D u_{h}\right\|_{L^{2}(\Omega)}=0 & \text { for } \gamma \in(2(1-s), 2 s), \\
\left\|u-u_{h}\right\|_{W^{1,2}(\Omega)} \leqslant c_{5} h^{s-1 / 2} & \text { for } \gamma=1 .
\end{array}
$$

The constant $c_{5}>0$ depends on $c_{1}, c_{2}$, and upper bounds for $\|u\|_{H^{1+s}(\Omega)},\left|u_{h}\right|_{W^{1, p}(\Omega)},|u|_{W^{1, p}(\Omega)}$. 
Proof. Proposition 2.2 and Theorem 2.1 prove

$\|e\|_{L^{2}(\Omega)}^{2}+h^{\gamma}\|D e\|_{L^{2}(\Omega)}^{2} \lesssim\left|e-e_{h}\right|_{W^{1, p}(\Omega)}^{r /(r-1)}+\left\|e-e_{h}\right\|_{L^{2}(\Omega)}^{2}+h^{\gamma}\left\|D\left(e-e_{h}\right)\right\|_{L^{2}(\Omega)}^{2}+a_{h}\left(u, e_{h}\right)$

for the nodal interpolant $e_{h} \in \mathcal{S}_{0}^{1}(\mathcal{T})^{m}$ of $e \in C\left(\bar{\Omega} ; \mathbb{R}^{m}\right)$. Standard estimates on nodal interpolation in $H^{1+s}(\Omega)$ and $r /(r-1) \geqslant 2$ imply

$$
\left|e-e_{h}\right|_{W^{1, p}(\Omega)}^{r /(r-1)}+\left\|e-e_{h}\right\|_{L^{2}(\Omega)}^{2}+h^{\gamma}\left\|D\left(e-e_{h}\right)\right\|_{L^{2}(\Omega)}^{2} \lesssim h^{2 s}+h^{2+2 s}+h^{\gamma+2 s} .
$$

If $u \in H^{2}\left(\Omega ; \mathbb{R}^{m}\right)$ then integration by parts and $e_{h}=0$ on $\partial \Omega$ show

$$
h^{\gamma} \int_{\Omega} D u: D e_{h} \mathrm{~d} x \leqslant h^{\gamma}\|u\|_{H^{2}(\Omega)}\left\|e_{h}\right\|_{L^{2}(\Omega)} .
$$

Hölder's inequality and an elementwise inverse estimate imply

$$
h^{\gamma} \int_{\Omega} D u: D e_{h} \mathrm{~d} x \lesssim h^{\gamma-1}\|u\|_{H^{1}(\Omega)}\left\|e_{h}\right\|_{L^{2}(\Omega)} .
$$

Interpolation of the last two estimates yields

$$
a_{h}\left(u, e_{h}\right)=h^{\gamma} \int_{\Omega} D u: D e_{h} \mathrm{~d} x \lesssim h^{\gamma-(1-s)}\|u\|_{H^{1+s}(\Omega)}\left\|e_{h}\right\|_{L^{2}(\Omega)} .
$$

We further estimate

$$
\begin{aligned}
a_{h}\left(u, e_{h}\right) & \lesssim h^{\gamma-(1-s)}\|u\|_{H^{1+s}(\Omega)}\left\|e_{h}\right\|_{L^{2}(\Omega)} \\
& \leqslant h^{\gamma-(1-s)}\|u\|_{H^{1+s}(\Omega)}\left\|e-e_{h}\right\|_{L^{2}(\Omega)}+h^{\gamma-(1-s)}\|u\|_{H^{1+s}(\Omega)}\|e\|_{L^{2}(\Omega)} .
\end{aligned}
$$

Nodal interpolation estimates and Young's inequality imply that for $\varrho>0$,

$$
a_{h}\left(u, e_{h}\right) \lesssim h^{\gamma-(1-s)+1+s}+h^{2 \gamma-2(1-s)}+\varrho\|e\|_{L^{2}(\Omega)}^{2} .
$$

The combination with 8.2 shows, after absorbing $\|e\|_{L^{2}(\Omega)}$ on the right-hand side,

$$
\|e\|_{L^{2}(\Omega)}^{2}+h^{\gamma}\|D e\|_{L^{2}(\Omega)}^{2} \lesssim h^{2 s}+h^{2 \gamma-2(1-s)} .
$$

The following theorem states that the stabilisation scheme $[8.1]$ is in fact the scheme of [NW] in 1D (up to a lumped integration of the right-hand side $f$ ).

THEOREM 8.2 Let $n=m=1, \Omega:=(0,1), \mathcal{A}=\mathcal{A}_{D}:=W_{0}^{1, p}(0,1)$,

$$
J(u ; v):=\int_{0}^{1} u v \mathrm{~d} x, \quad J_{h}\left(u_{h} ; v_{h}\right):=\frac{1}{2} \sum_{z \in \mathcal{K}} h_{z} u_{h}(z) v_{h}(z)
$$

for $u, v \in W_{0}^{1, p}(0,1)$ and $u_{h}, v_{h} \in \mathcal{A}_{h}=\mathcal{A}_{D, h}:=\mathcal{S}_{0}^{1}(\mathcal{T})$. Then, for all $u_{h}, v_{h} \in \mathcal{A}_{h}$,

$$
J_{h}\left(u_{h} ; v_{h}\right)=J\left(u_{h} ; v_{h}\right)+\frac{1}{6} \int_{0}^{1} h_{\mathcal{T}}^{2} D u_{h} D v_{h} \mathrm{~d} x
$$


Proof. Let $0=z_{0}<z_{1}<\cdots<z_{m+1}=1$ be such that $\mathcal{N}=\left\{z_{0}, z_{1}, \cdots, z_{m+1}\right\}$ and set $h_{j}:=z_{j}-z_{j-1}$ for $j=1, \ldots, m+1$ so that $h_{z_{j}}=h_{j}+h_{j+1}$ for $j=1, \ldots, m$. Elementary calculations with $v_{h}\left(z_{0}\right)=v_{h}\left(z_{m+1}\right)=0$ show

$$
\begin{aligned}
J\left(u_{h} ; v_{h}\right)= & \frac{1}{6} \sum_{j=1}^{m+1} h_{j}\left(2 u_{h}\left(z_{j-1}\right) v_{h}\left(z_{j-1}\right)+u_{h}\left(z_{j-1}\right) v_{h}\left(z_{j}\right)\right. \\
& \left.+u_{h}\left(z_{j}\right) v_{h}\left(z_{j-1}\right)+2 u_{h}\left(z_{j}\right) v_{h}\left(z_{j}\right)\right), \\
J_{h}\left(u_{h} ; v_{h}\right)= & \frac{1}{2} \sum_{j=1}^{m+1} h_{j}\left(u_{h}\left(z_{j-1}\right) v_{h}\left(z_{j-1}\right)+u_{h}\left(z_{j}\right) v_{h}\left(z_{j}\right)\right) .
\end{aligned}
$$

Hence,

$$
\begin{aligned}
J_{h}\left(u_{h} ; v_{h}\right)-J\left(u_{h} ; v_{h}\right)= & \frac{1}{6} \sum_{j=1}^{m+1} h_{j}\left(u_{h}\left(z_{j-1}\right) v_{h}\left(z_{j-1}\right)-u_{h}\left(z_{j-1}\right) v_{h}\left(z_{j}\right)\right. \\
& \left.-u_{h}\left(z_{j}\right) v_{h}\left(z_{j-1}\right)+u_{h}\left(z_{j}\right) v_{h}\left(z_{j}\right)\right) \\
= & \frac{1}{6} \sum_{j=1}^{m+1} h_{j}\left(u_{h}\left(z_{j}\right)-u_{h}\left(z_{j-1}\right)\right)\left(v_{h}\left(z_{j}\right)-v_{h}\left(z_{j-1}\right)\right) \\
= & \frac{1}{6} \int_{0}^{1} h_{\mathcal{T}}^{2} D u_{h} D v_{h} \mathrm{~d} x .
\end{aligned}
$$

The parameter $\gamma=2$ is critical in Theorem 8.1 and excluded in our analysis. In fact, the arguments in $[\mathrm{NW}]$ are quite different and restricted to a model scenario in 1D.

\section{Strong convergence in the scalar 2-well problem}

In the case of the 2-well energy from Example 3.3 and $n \geqslant 2, m=1$ we can weaken (H4), i.e. the uniform monotonicity of $J$ can in fact be replaced by monotonicity.

(H5) There exists $B \geqslant 0$ such that, for $v \in W^{1, p}(\Omega)$,

$$
0 \leqslant J(u ; e)-J\left(u_{h} ; e\right), \quad J(u ; v)-J\left(u_{h} ; v\right) \leqslant B\|e\|_{L^{2}(\Omega)}\|v\|_{L^{2}(\Omega)} .
$$

We suppose that $J_{h}:=J+a_{h}$ with $a_{h}$ as in 6.1], 7.1], or 8.1.

THEOREM 9.1 Suppose $n \geqslant 2$ and $m=1$. Let $S=D \varphi$ with $\varphi$ as in Example 3.3 . Suppose (H5) holds and $u_{D} \in H^{2}\left(\mathcal{E}_{D} ; \mathbb{R}\right)$. Assume that $\mathcal{T}$ is quasiuniform and $u \in H^{2}(\Omega) \cap W^{1, p}(\Omega)$. Then

$$
\left\|u-u_{h}\right\|_{W^{1,2}(\Omega)} \leqslant c_{6} h^{1 / 2} \text { for } \gamma=1 .
$$

The constant $c_{6}>0$ depends on $c_{1}, c_{2}$, and upper bounds for $\|u\|_{H^{2}(\Omega)},\left|u_{h}\right|_{W^{1, p}(\Omega)},|u|_{W^{1, p}(\Omega)}$, and $\left\|\partial_{\mathcal{E}}^{2} u_{D} / \partial s^{2}\right\|_{L^{2}(\partial \Omega)}$.

The proof of the theorem follows from the following lemma and the estimates of the previous sections. 
LEMMA 9.1 Let $n \geqslant 2$ and let $\varphi$ be as in Example 3.3 and $S=D \varphi$. Suppose $e_{h} \in H^{1}(\Omega)$ satisfies $e_{h}=0$ on $\partial \Omega$. Then

$$
\|e\|_{L^{2}(\Omega)}^{2} \lesssim \int_{\Omega} \delta: D e \mathrm{~d} x+\left\|e-e_{h}\right\|_{L^{2}(\Omega)}^{2}+\left\|D\left(e-e_{h}\right)\right\|_{L^{2}(\Omega)}^{2} .
$$

Proof. Proposition 3 in [CP1] ensures the existence of some $a \in \mathbb{R}^{n}$ with $|a|=1$ such that

$$
\|a \cdot D e\|_{L^{2}(\Omega)}^{2} \lesssim \int_{\Omega} \delta \cdot D e \mathrm{~d} x
$$

A fine version of the Friedrichs inequality (which follows from the one-dimensional Friedrichs inequality) proves

$$
\left\|e_{h}\right\|_{L^{2}(\Omega)} \lesssim\left\|a \cdot D e_{h}\right\|_{L^{2}(\Omega)} .
$$

Two applications of the triangle inequality and the last two estimates prove the lemma.

Proof of Theorem 9.1 Proposition 2 and Theorem 2 in [CP1] prove (H1)-(H2). Setting $\|v\|_{X_{h}}^{2}=$ $\|v\|_{Y_{h}}^{2}:=a_{h}(v, v)$ we observe that the first estimate in (H3) is satisfied. Instead of the second estimate in (H3) we have

$$
J_{h}(u ; v)-J_{h}\left(u_{h} ; v\right) \leqslant\|e\|_{L^{2}(\Omega)}\|v\|_{L^{2}(\Omega)}+\|e\|_{X_{h}}\|v\|_{Y_{h}}
$$

for all $v \in Y_{h}$. This and Lemma 9.1 imply the estimate of Theorem 2.1. Hence,

$$
\begin{aligned}
\|e\|_{L^{2}(\Omega)}^{2}+a_{h}(e, e) \lesssim & \left|e-e_{h}\right|_{W^{1, p}(\Omega)}^{r /(r-1)}+\left\|e-e_{h}\right\|_{L^{2}(\Omega)}^{2} \\
& +\left\|D\left(e-e_{h}\right)\right\|_{L^{2}(\Omega)}^{2}+a_{h}\left(e-e_{h}, e-e_{h}\right)+2 a_{h}\left(u, e_{h}\right)
\end{aligned}
$$

for $e_{h} \in \mathcal{S}_{0}^{1}(\mathcal{T})$. The estimate of the theorem then follows as in the proofs of Theorem 6.1, 7.1, and 8.1 for $a_{h}$ defined by 6.1, 7.1, and 8.1, respectively.

\section{Acknowledgements}

The support by the DFG through the priority program 1095 "Analysis, Modelling and Simulation of Multiscale Problems" is thankfully acknowledged. This work was initiated when all authors were members or guests at the Graduiertenkolleg 357 "Efficient Algorithms and Multiscale Problems" in Kiel, Germany, continued at the Vienna University of Technology, Austria, and finished while all the authors enjoyed the hospitality of the Newton Institute, Cambridge, England, UK, during the programme Computational Challenges in Partial Differential Equations.

\section{REFERENCES}

[Ba] BARTELS, S. Numerical analysis of some non-convex variational problems. PhD thesis, Christian Albrechts-University, Kiel (2001). Available at http://e-diss.uni-kiel.de/math-nat.html.

[BP] BARTELS, S. \& PROHL, A. Multiscale resolution in the computation of crystalline microstructure. Numer. Math. 96 (2004), 641-660. 
[BS] Brenner, S. C. \& Scott, L. R. The Mathematical Theory of Finite Element Methods. Texts in Appl. Math. 15, Springer (1994). Zbl 0804.65101 MR 95f:65001

[Bo] BolzA, O. A fifth necessary condition for a strong extremum of the integral $\int_{x_{0}}^{x_{1}} f\left(x, y, y^{\prime}\right) \mathrm{d} x$. Trans. Amer. Math. Soc. 7 (1906), 314-324. Zbl 37.0397.01(JFM 37.0397.01) MR 1500751

[C] CARSTENSEN, C. (2002), All first order averaging techniques yield reliable and efficient a posteriori finite element error control. Math. Comp. (to appear).

[CK] Carstensen, C. \& Klose, R. Guaranteed a posteriori finite element error control for the $p$-Laplace problem. Preprint (2002).

[CM] CARSTENSEN, C. \& MÜLLER, S. Local stress regularity in scalar nonconvex variational problems. SIAM J. Math. Anal. 34 (2002), 495-509. Zbl 1012.49027 | MR 2003k:49075

[CP1] Carstensen, C. \& Plecháč, P. Numerical solution of the scalar double-well problem allowing microstructure. Math. Comp. 66 (1997), 997-1026. Zbl 0870.65055 MR 97i:65163

[CP2] Carstensen, C. \& PlecháČ, P. Numerical analysis of compatible phase transitions in elastic solids. SIAM J. Numer. Anal. 37 (2000), 2061-2081. Zbl pre01519664 MR 2001i:74066

[CP3] CARstensen, C. \& PLeChÁČ, P. Numerical analysis of a relaxed variational model of hysteresis in two-phase solids. M2AN Math. Model. Numer. Anal. 35 (2001), 865-878. Zbl 1007.74062 MR 2003c:74071

[F] FRENCH, D. A. On the convergence of finite element approximations of a relaxed variational problem. SIAM J. Numer. Anal. 27 (1990), 419-436. Zbl 0696.65070 MR 91f:65167

[GKR] Goodman, J. \& Kohn, R., REYnA, L. A numerical study of a relaxed variational problem from optimal design. Comput. Methods Appl. Engrg. 57 (1986), 107-127. Zbl 0591.73119 MR 88a:73054

[K] Kohn, R. V. The relaxation of a double-well energy. Contin. Mech. Thermodyn. 3 (1991), 193-236. Zbl 0825.73029 MR 93d:73014

[LB] LiU, W. B. \& BARRETT, J. W. A remark on the regularity of the solutions of the $p$-Laplacian and its application to their finite element approximation. J. Math. Anal. Appl. 178 (1993), 470-487. Zbl 0799.35085 MR 95a:35016

[L1] LUSKIN, M. On the computation of crystalline microstructure. Acta Numer. 5 (1996), 191-257. Zbl 0867.65033 MR 99f:73030

[L2] Luskin, M. Approximation of a laminated microstructure for a rotational invariant double-well energy density. Numer. Math. 75 (1996), 205-221. Zbl 0874.73060 MR 97k:73026

[NW] Nicolaides, R. A. \& Walkington, N. J. Strong convergence of numerical solutions to degenerate variational problems. Math. Comp. 64 (1995), 117-127. Zbl 0821.65040 MR 95m:65183

[P] Prohl, A. Computational Micromagnetism. Adv. Numer. Math., Teubner (2001).

[R] RoubíČEK, T. Relaxation in Optimization Theory and Variational Calculus. De Gruyter Ser. Nonlinear Anal. Appl. 4, de Gruyter, New York (1997). Zbl 0880.49002 MR 98e:49002

[Y] Young, L. C. Generalized curves and the existence of an attained absolute minimum in the calculus of variations. C. R. Soc. Sciences Lettres Varsovie Cl. III 30 (1937), 212-234. Z Zbl 0019.21901 